\title{
PARTICIPATION OF FARM WOMEN ON THEIR RELATIVE EMPOWERMENT IN SELF DECISIONS IN RURAL AREAS OF TELANGANA, INDIA
}

\section{B. PRASHANTHI ${ }^{1 *}$, DR. M. SARADA DEVI ${ }^{2 *}$, DR. P. SREEDEVI ${ }^{3 *}$, DR. R. NEELA RANI ${ }^{4 *} \&$ DR. S. SUCHIRITHA DEVI ${ }^{5 *}$}

${ }^{I}$ Ph.D Research Scholar, Department of Human Development and Family Studies, College of Community Science, Professor Jayashankar Telangana State Agricultural University, Hyderabad, Telangana, India

${ }^{2}$ Professor and University Head, Department of Human Development and Family Studies, College of Community Science, Professor Jayashankar Telangana State Agricultural University, Hyderabad, Telangana, India

${ }^{3}$ Assistant Professor, Department of Human Development and Family Studies, College of Community Science,

Professor Jayashankar Telangana State Agricultural University, Hyderabad, Telangana, India

${ }^{4}$ Principal Scientist, Department of Extension Education and Communication Management, AICRP-WIA, PG\&RC

Professor Jayashankar Telangana State Agricultural University, Hyderabad, Telangana, India

${ }^{5}$ Associate professor, Department of Food and Nutrition, College of Community Science, PG\&RC

Professor Jayashankar Telangana State Agricultural University, Hyderabad, Telangana, India

\section{ABSTRACT}

Women empowerment is giving legitimate power or authority to perform the tasks. If women were empowered they would be able to participate in the planning and decision making tasks, and contribute to the development programmes and activities individually. The participation of women in work and social life has put heavy demand upon them to make adjustments between their traditional role in their families and outside their home. The study was conducted on 120 farm women from rural areas of Telangana, in-depth interview method was used to collect the data from the respondents. Empowerment scale was used as tool to collect the data from the sample. In all the self decisions related to personal, children and household affairs, farm women were disempowered suppressed by the society. Lack of education and low levels of decision making skills in all the aspects related to empowerment such as resources available, access to resources, decision making and action taking, resulted in disempowered women.

KEYWORDS: Empowerment, self decisions, personal affairs, children affairs, household affairs. Farm women, rural areas. Education. Life skills

Received: Feb 18, 2021; Accepted: Mar 08, 2021; Published: Mar 13, 2021; Paper Id.: IJASRAPR20217

\section{INTRODUCTION}

Women are the backbone of agricultural workforce but worldwide their hard work has mostly been unpaid. They do the most tedious and back-breaking tasks in agriculture, animal husbandry and in homes. Due to various problems that are being faced by the women farmers, they are not able to contribute more to the agriculture of our country. The major obstacles start at their homes in the form of gender inequality, not able to decide by themselves in farm activities and lack of awareness about the latest technologies of agriculture. When a women empowers themselves in agriculture, the nation starts developing faster and more widely. In extension activities the women is now the centre point and active. This empowerment initiates a global level support for humanists thinking. Her enlightenment will change the face of rural India. 
Women's empowerment is a process in which women get power or authority to challenge certain situations (Basu and Basu, 2001). Empowerment of women is a prerequisite and a necessary requirement in order to make the process of personal development and development of a country stable and sustainable; and it generally takes place when women challenge the prevailing customs and culture to improve their well-being. The campaign for women's empowerment has two-fold objectives: a. Social justice, a significant facet of human well-being, which is inherently worth following and b. It's a means to additional ends (Sridevi, 2005).

\section{Operational Definitions}

Self Decisions: The decisions that were exclusively taken by the women.

Personal Affairs: Aspects of decisions related to only self of the women.

Children's Affairs: Aspects of decisions related to children's

House hold Affairs: Aspects of decisions related to general household.

Resources Available: Resources available to the family for a particular task.

Access to resources: Women's access to resources in the family to person a particular task.

Decision making: Women's decision making capacity for a particular task to be achieved.

Action taken: Women's action in getting a particular task done.

\section{Studies Related to Empowerment}

Raazia Hassan Naqvi and Muhammad Ibrar (2015) elicited that in majority of the rural family most of the decisions regarding household and agricultural matter are taken up by the family head alone. Wife is consulted for only households matter and not being involved for taking independent or joint decision in the agricultural matters. In a negligible proportion, the wife takes independent decisions.

Kavita Baliyan (2014) observed that women's role was found prominent in petty household issues only. In most of the cases women acted as initiators in deciding about some major household affairs but final decisions were being taken ultimately by men. In rural India, even though women participate in economic activities, they have little role in decision making, particularly in matters related to agriculture and financial matters.

Sabina Bano (2014) stated that participation of women in decision making is likely to be affected by socioeconomic status of women. Gender differences led women to suppress in the decision making on various issues occurring in their daily activities. Roles and responsibilities of women and their choices are restricted to specific sphere.

Sharma Rao and Sharma (2013) research stated that men dominated in majority of the farm decisions in rural families. Decisions related to buying and selling of land, machines and other agricultural implements; improvements of harvest and livestock management were mainly taken by head of the family or husbands.

Gupta and Yesudian (2006) considered four dimensions of women's empowerment: household autonomy, mobility, and attitudes toward gender and towards domestic violence.

Devi and Rayalu (2003) findings suggested that working women perceived higher levels of empowerment in all the aspects related to self-decision than non-working women. 
Minaxi and Lopamudra (2000) showed that women are considered neither knowledgeable nor competent enough to participate in the process of decision making. Major decisions about farming are mainly taken by the male family head. The influence of women in decision making about farming affairs was found to be only supportive in nature.

Zaman, et al. (2000) find out the level of empowerment of women in household decision making process in some purposively selected rural and urban areas of Bangladesh. It has considered 18 variables in household domain, namely, freedom of purchase saris, freedom of purchasing cosmetics, opinion for children's admission in school, opinion seeks for child birth, opinion for family planning, freedom of purchase children's clothing, treatment autonomy for children, treatment autonomy for own, freedom for expenditure, knowledge about inheritance law, freedom of expenditure for own, freedom of saving money, freedom of purchasing ornaments, free to move outside, opinion for land dispute, freedom to travel, freedom to purchase of properties for own and whether a microcredit holder. Using these variables, a composite level of women's empowerment was assessed by Principle Component Analysis. After getting the score, they categorized empowerment as poor with score 1-82, fair with score 83-122 and good with score above 123. The authors showed that $40 \%$ had poor, $19 \%$ had fair and $41 \%$ had good level of women's empowerment. Using bivariate analysis this study has revealed that level of women's empowerment is high among the respondents living in the urban areas, having less number of children, religion in Islam, having higher level of education of both husband and wife, engaged in jobs, having higher monthly income contributed to family and living in pacca house $(\mathrm{p}<0.05)$. It also shows that there are no significant association with current age of both husband and wife, age at marriage and spousal age difference ( $p>0.05)$. This study does not clarify how the scores were given to assess empowerment and it considers only individual factors at household level.

\section{MATERIALS AND METHODS}

The present study was conducted in rural areas of Telangana State by using an experimental research design. A total of 120 farm women were selected by purposive sampling technique. Data was collected by administering standardized empowerment scale which collects information regarding self decisions of women in their personal, child and house hold affairs. 


\section{RESULTS AND DISCUSSION}

Table 1: Percentage Distribution of Respondents According to Self Decisions on Personal Affairs.

\begin{tabular}{|c|c|c|c|c|c|c|c|c|c|c|c|c|c|}
\hline \multirow{2}{*}{$\begin{array}{l}\text { S. } \\
\text { No. }\end{array}$} & \multirow{2}{*}{ Items } & \multicolumn{3}{|c|}{ Resource Available } & \multicolumn{3}{|c|}{ Access to resource } & \multicolumn{3}{|c|}{ Decision making } & \multicolumn{3}{|c|}{ Action } \\
\hline & & A & $\mathrm{S}$ & $\mathrm{N}$ & $\mathrm{A}$ & $\mathrm{S}$ & $\mathrm{N}$ & A & $\mathrm{S}$ & $\mathrm{N}$ & A & $\mathrm{S}$ & $\mathrm{N}$ \\
\hline 1 & $\begin{array}{l}\text { Freedom to pursue own } \\
\text { hobbies }\end{array}$ & $\begin{array}{c}6 \\
(15 \% \\
)\end{array}$ & $\begin{array}{c}68 \\
(57 \%)\end{array}$ & $\begin{array}{c}46 \\
(38 \%)\end{array}$ & $\begin{array}{c}7 \\
(6 \%)\end{array}$ & $\begin{array}{c}71 \\
(59 \%)\end{array}$ & $\begin{array}{c}42 \\
(35 \%)\end{array}$ & $\begin{array}{c}3 \\
(3 \%)\end{array}$ & $\begin{array}{c}71 \\
(59 \%)\end{array}$ & $\begin{array}{c}46 \\
(38 \%)\end{array}$ & $\begin{array}{c}2 \\
(2 \%)\end{array}$ & $\begin{array}{c}69 \\
(58 \%)\end{array}$ & $\begin{array}{c}49 \\
(41 \%)\end{array}$ \\
\hline 2 & $\begin{array}{l}\text { Freedom to pursue own } \\
\text { religious activities }\end{array}$ & $\begin{array}{c}7 \\
(6 \%)\end{array}$ & $\begin{array}{c}89 \\
(74 \%)\end{array}$ & $\begin{array}{c}24 \\
(20 \%)\end{array}$ & $\begin{array}{c}5 \\
(4 \%)\end{array}$ & $\begin{array}{c}91 \\
(76 \%)\end{array}$ & $\begin{array}{c}24 \\
(20 \%)\end{array}$ & $\begin{array}{c}6 \\
(5 \%)\end{array}$ & $\begin{array}{c}90 \\
(75 \%)\end{array}$ & $\begin{array}{c}24 \\
(20 \%)\end{array}$ & $\begin{array}{c}3 \\
(3 \%)\end{array}$ & $\begin{array}{c}88 \\
(73 \%)\end{array}$ & $\begin{array}{c}29 \\
(24 \%)\end{array}$ \\
\hline 3 & $\begin{array}{l}\text { Personal movement to } \\
\text { shorter distance }\end{array}$ & $\begin{array}{c}4 \\
(3 \%)\end{array}$ & $\begin{array}{c}77 \\
(64 \%)\end{array}$ & $\begin{array}{c}39 \\
(33 \%)\end{array}$ & $\begin{array}{c}3 \\
(3 \%)\end{array}$ & $\begin{array}{c}78 \\
(65 \%)\end{array}$ & $\begin{array}{c}39 \\
(33 \%)\end{array}$ & $\begin{array}{c}6 \\
(5 \%)\end{array}$ & $\begin{array}{c}71 \\
(59 \%)\end{array}$ & $\begin{array}{c}43 \\
(36 \%)\end{array}$ & $\begin{array}{c}7 \\
(6 \%)\end{array}$ & $\begin{array}{c}74 \\
(62 \%)\end{array}$ & $\begin{array}{c}39 \\
(33 \%)\end{array}$ \\
\hline 4 & $\begin{array}{l}\text { Personal Movement to } \\
\text { longer distances }\end{array}$ & $\begin{array}{c}7 \\
(6 \%)\end{array}$ & $\begin{array}{c}43 \\
(36 \%)\end{array}$ & $\begin{array}{c}70 \\
(58 \%)\end{array}$ & $\begin{array}{c}3 \\
(3 \%)\end{array}$ & $\begin{array}{c}42 \\
(35 \%)\end{array}$ & $\begin{array}{c}75 \\
(63 \%)\end{array}$ & $\begin{array}{c}5 \\
(4 \%) \\
\end{array}$ & $\begin{array}{c}36 \\
(30 \%)\end{array}$ & $\begin{array}{c}79 \\
(66 \%)\end{array}$ & $\begin{array}{c}2 \\
(2 \%)\end{array}$ & $\begin{array}{c}39 \\
(33 \%)\end{array}$ & $\begin{array}{c}79 \\
(66 \%)\end{array}$ \\
\hline 5 & Buying sarees/dresses & $\begin{array}{c}3 \\
(3 \%)\end{array}$ & $\begin{array}{c}32 \\
(27 \%)\end{array}$ & $\begin{array}{c}85 \\
(71 \%)\end{array}$ & $\begin{array}{c}6 \\
(5 \%)\end{array}$ & $\begin{array}{c}49 \\
(41 \%)\end{array}$ & $\begin{array}{c}65 \\
(54 \%)\end{array}$ & $\begin{array}{c}5 \\
(4 \%) \\
\end{array}$ & $\begin{array}{c}38 \\
(32 \%)\end{array}$ & $\begin{array}{c}77 \\
(64 \%)\end{array}$ & $\begin{array}{c}2 \\
(2 \%)\end{array}$ & $\begin{array}{c}52 \\
(43 \%) \\
\end{array}$ & $\begin{array}{c}66 \\
(55 \%)\end{array}$ \\
\hline 6 & $\begin{array}{l}\text { Buying less expensive } \\
\text { jewellery }\end{array}$ & $\begin{array}{c}7 \\
(6 \%)\end{array}$ & $\begin{array}{c}64 \\
(53 \%)\end{array}$ & $\begin{array}{c}49 \\
(41 \%)\end{array}$ & $\begin{array}{c}7 \\
(6 \%)\end{array}$ & $\begin{array}{c}61 \\
(51 \%)\end{array}$ & $\begin{array}{c}52 \\
(43 \%)\end{array}$ & $\begin{array}{c}4 \\
(3 \%)\end{array}$ & $\begin{array}{c}68 \\
(57 \%)\end{array}$ & $\begin{array}{c}48 \\
(40 \%)\end{array}$ & $\begin{array}{c}5 \\
(4 \%)\end{array}$ & $\begin{array}{c}63 \\
(53 \%)\end{array}$ & $\begin{array}{c}52 \\
(43 \%)\end{array}$ \\
\hline 7 & $\begin{array}{l}\text { Buying expensive } \\
\text { jewellery }\end{array}$ & $\begin{array}{c}4 \\
(3 \%)\end{array}$ & $\begin{array}{c}65 \\
(54 \%)\end{array}$ & $\begin{array}{c}51 \\
(43 \%)\end{array}$ & $\begin{array}{c}5 \\
(4 \%) \\
\end{array}$ & $\begin{array}{c}68 \\
(57 \%)\end{array}$ & $\begin{array}{c}47 \\
(39 \%)\end{array}$ & $\begin{array}{c}7 \\
(6 \%)\end{array}$ & $\begin{array}{c}68 \\
(57 \%)\end{array}$ & $\begin{array}{c}45 \\
(38 \%)\end{array}$ & $\begin{array}{c}4 \\
(3 \%)\end{array}$ & $\begin{array}{c}69 \\
(58 \%) \\
\end{array}$ & $\begin{array}{c}47 \\
(39 \%)\end{array}$ \\
\hline 8 & $\begin{array}{l}\text { Freedom to express } \\
\text { emotions freely }\end{array}$ & $\begin{array}{c}4 \\
(3 \%) \\
\end{array}$ & $\begin{array}{c}60 \\
(50 \%) \\
\end{array}$ & $\begin{array}{c}56 \\
(47 \%) \\
\end{array}$ & $\begin{array}{c}3 \\
(3 \%) \\
\end{array}$ & $\begin{array}{c}57 \\
(48 \%) \\
\end{array}$ & $\begin{array}{c}60 \\
(50 \%) \\
\end{array}$ & $\begin{array}{c}6 \\
(5 \%) \\
\end{array}$ & $\begin{array}{c}64 \\
(53 \%) \\
\end{array}$ & $\begin{array}{c}50 \\
(42 \%) \\
\end{array}$ & $\begin{array}{c}3 \\
(3 \%) \\
\end{array}$ & $\begin{array}{c}62 \\
(52 \%) \\
\end{array}$ & $\begin{array}{c}55 \\
(46 \%) \\
\end{array}$ \\
\hline 9 & $\begin{array}{l}\text { Freedom to project own } \\
\text { personality in a group }\end{array}$ & $\begin{array}{c}5 \\
(4 \%) \\
\end{array}$ & $\begin{array}{c}56 \\
(47 \%) \\
\end{array}$ & $\begin{array}{c}59 \\
(49 \%) \\
\end{array}$ & $\begin{array}{c}3 \\
(3 \%) \\
\end{array}$ & $\begin{array}{c}58 \\
(48 \%) \\
\end{array}$ & $\begin{array}{c}59 \\
(49 \%) \\
\end{array}$ & $\begin{array}{c}3 \\
(3 \%) \\
\end{array}$ & $\begin{array}{c}67 \\
(56 \%) \\
\end{array}$ & $\begin{array}{c}50 \\
(42 \%) \\
\end{array}$ & $\begin{array}{c}3 \\
(3 \%) \\
\end{array}$ & $\begin{array}{c}66 \\
(55 \%) \\
\end{array}$ & $\begin{array}{c}51 \\
(43 \%) \\
\end{array}$ \\
\hline 10 & $\begin{array}{l}\text { Planning for no. of } \\
\text { children }\end{array}$ & $\begin{array}{c}6 \\
(5 \%) \\
\end{array}$ & $\begin{array}{c}62 \\
(52 \%) \\
\end{array}$ & $\begin{array}{c}52 \\
(43 \%) \\
\end{array}$ & $\begin{array}{c}4 \\
(3 \%) \\
\end{array}$ & $\begin{array}{c}67 \\
(56 \%) \\
\end{array}$ & $\begin{array}{c}49 \\
(41 \%) \\
\end{array}$ & $\begin{array}{c}1 \\
(1 \%)\end{array}$ & $\begin{array}{c}55 \\
(46 \%) \\
\end{array}$ & $\begin{array}{c}64 \\
(53 \%) \\
\end{array}$ & $\begin{array}{c}2 \\
(2 \%) \\
\end{array}$ & $\begin{array}{c}62 \\
(52 \%) \\
\end{array}$ & $\begin{array}{c}56 \\
(47 \%) \\
\end{array}$ \\
\hline 11 & Spacing the children & $\begin{array}{c}7 \\
(6 \%) \\
\end{array}$ & $\begin{array}{c}60 \\
(50 \%) \\
\end{array}$ & $\begin{array}{c}53 \\
(44 \%) \\
\end{array}$ & $\begin{array}{c}2 \\
(2 \%) \\
\end{array}$ & $\begin{array}{c}59 \\
(49 \%) \\
\end{array}$ & $\begin{array}{c}59 \\
(49 \%) \\
\end{array}$ & $\begin{array}{c}7 \\
(6 \%) \\
\end{array}$ & $\begin{array}{c}56 \\
(47 \%) \\
\end{array}$ & $\begin{array}{c}57 \\
(48 \%) \\
\end{array}$ & $\begin{array}{c}3 \\
(3 \%) \\
\end{array}$ & $\begin{array}{c}61 \\
(51 \%) \\
\end{array}$ & $\begin{array}{c}56 \\
(47 \%) \\
\end{array}$ \\
\hline 12 & $\begin{array}{l}\text { Freedom to use } \\
\text { contraceptive according to } \\
\text { own choice }\end{array}$ & $\begin{array}{c}5 \\
(4 \%)\end{array}$ & $\begin{array}{c}59 \\
(49 \%)\end{array}$ & $\begin{array}{c}56 \\
(47 \%)\end{array}$ & $\begin{array}{c}2 \\
(2 \%)\end{array}$ & $\begin{array}{c}59 \\
(49 \%)\end{array}$ & $\begin{array}{c}59 \\
(49 \%)\end{array}$ & $\begin{array}{c}2 \\
(2 \%)\end{array}$ & $\begin{array}{c}70 \\
(58 \%)\end{array}$ & $\begin{array}{c}48 \\
(40 \%)\end{array}$ & $\begin{array}{c}1 \\
(1 \%)\end{array}$ & $\begin{array}{c}60 \\
(50 \%)\end{array}$ & $\begin{array}{c}59 \\
(49 \%)\end{array}$ \\
\hline 13 & $\begin{array}{l}\text { Freedom to spend own } \\
\text { money }\end{array}$ & $\begin{array}{c}4 \\
(3 \%)\end{array}$ & $\begin{array}{c}54 \\
(45 \%) \\
\end{array}$ & $\begin{array}{c}62 \\
(52 \%) \\
\end{array}$ & $\begin{array}{c}4 \\
(3 \%) \\
\end{array}$ & $\begin{array}{c}58 \\
(48 \%) \\
\end{array}$ & $\begin{array}{c}58 \\
(48 \%) \\
\end{array}$ & $\begin{array}{c}3 \\
(3 \%)\end{array}$ & $\begin{array}{c}57 \\
(48 \%) \\
\end{array}$ & $\begin{array}{c}60 \\
(50 \%) \\
\end{array}$ & $\begin{array}{c}3 \\
(3 \%)\end{array}$ & $\begin{array}{c}56 \\
(47 \%) \\
\end{array}$ & $\begin{array}{c}61 \\
(51 \%)\end{array}$ \\
\hline 14 & Inviting friends home & $\begin{array}{c}5 \\
(4 \%) \\
\end{array}$ & $\begin{array}{c}64 \\
(53 \%) \\
\end{array}$ & $\begin{array}{c}51 \\
(43 \%)\end{array}$ & $\begin{array}{c}4 \\
(3 \%) \\
\end{array}$ & $\begin{array}{c}68 \\
(57 \%) \\
\end{array}$ & $\begin{array}{c}48 \\
(40 \%)\end{array}$ & $\begin{array}{c}6 \\
(5 \%) \\
\end{array}$ & $\begin{array}{c}57 \\
(48 \%) \\
\end{array}$ & $\begin{array}{c}57 \\
(48 \%)\end{array}$ & $\begin{array}{c}3 \\
(3 \%)\end{array}$ & $\begin{array}{c}64 \\
(53 \%)\end{array}$ & $\begin{array}{c}53 \\
(44 \%) \\
\end{array}$ \\
\hline 15 & $\begin{array}{l}\text { Freedom to save money } \\
\text { according to own choice }\end{array}$ & $\begin{array}{c}4 \\
(3 \%)\end{array}$ & $\begin{array}{c}69 \\
(58 \%)\end{array}$ & $\begin{array}{c}47 \\
(39 \%)\end{array}$ & $\begin{array}{c}4 \\
(3 \%)\end{array}$ & $\begin{array}{c}59 \\
(49 \%)\end{array}$ & $\begin{array}{c}57 \\
(48 \%)\end{array}$ & $\begin{array}{c}3 \\
(3 \%)\end{array}$ & $\begin{array}{c}58 \\
(48 \%)\end{array}$ & $\begin{array}{c}59 \\
(49 \%)\end{array}$ & $\begin{array}{c}6 \\
(5 \%)\end{array}$ & $\begin{array}{c}61 \\
(51 \%)\end{array}$ & $\begin{array}{c}53 \\
(44 \%)\end{array}$ \\
\hline 16 & $\begin{array}{l}\text { Freedom to attend social } \\
\text { functions }\end{array}$ & $\begin{array}{c}4 \\
(3 \%) \\
\end{array}$ & $\begin{array}{c}63 \\
(53 \%) \\
\end{array}$ & $\begin{array}{c}53 \\
(44 \%) \\
\end{array}$ & -- & $\begin{array}{c}54 \\
(45 \%) \\
\end{array}$ & $\begin{array}{c}66 \\
(55 \%) \\
\end{array}$ & $\begin{array}{c}3 \\
(3 \%) \\
\end{array}$ & $\begin{array}{c}59 \\
(49 \%) \\
\end{array}$ & $\begin{array}{c}58 \\
(48 \%) \\
\end{array}$ & $\begin{array}{c}3 \\
(3 \%) \\
\end{array}$ & $\begin{array}{c}69 \\
(58 \%) \\
\end{array}$ & $\begin{array}{c}48 \\
(40 \%) \\
\end{array}$ \\
\hline 17 & $\begin{array}{l}\text { Have control of one's own } \\
\text { time }\end{array}$ & $\begin{array}{c}5 \\
(4 \%) \\
\end{array}$ & $\begin{array}{c}58 \\
(48 \%)\end{array}$ & $\begin{array}{c}57 \\
(48 \%)\end{array}$ & $\begin{array}{c}2 \\
(2 \%)\end{array}$ & $\begin{array}{c}65 \\
(54 \%)\end{array}$ & $\begin{array}{c}53 \\
(44 \%)\end{array}$ & $\begin{array}{c}5 \\
(4 \%)\end{array}$ & $\begin{array}{c}58 \\
(48 \%)\end{array}$ & $\begin{array}{c}57 \\
(48 \%)\end{array}$ & $\begin{array}{c}3 \\
(3 \%)\end{array}$ & $\begin{array}{c}70 \\
(58 \%)\end{array}$ & $\begin{array}{c}47 \\
(39 \%)\end{array}$ \\
\hline 18 & $\begin{array}{l}\text { Can do things according } \\
\text { to one's own interests }\end{array}$ & $\begin{array}{c}4 \\
(3 \%) \\
\end{array}$ & $\begin{array}{c}59 \\
(49 \%) \\
\end{array}$ & $\begin{array}{c}57 \\
(48 \%) \\
\end{array}$ & $\begin{array}{c}2 \\
(2 \%) \\
\end{array}$ & $\begin{array}{c}63 \\
(53 \%) \\
\end{array}$ & $\begin{array}{c}55 \\
(46 \%) \\
\end{array}$ & $\begin{array}{c}4 \\
(3 \%) \\
\end{array}$ & $\begin{array}{c}62 \\
(52 \%) \\
\end{array}$ & $\begin{array}{c}54 \\
(45 \%) \\
\end{array}$ & $\begin{array}{c}4 \\
(3 \%) \\
\end{array}$ & $\begin{array}{c}57 \\
(48 \%) \\
\end{array}$ & $\begin{array}{c}59 \\
(49 \% \\
\end{array}$ \\
\hline 19 & $\begin{array}{l}\text { Freedom to concentrate } \\
\text { on office work/personal } \\
\text { work wholly }\end{array}$ & $\begin{array}{c}4 \\
(3 \%)\end{array}$ & $\begin{array}{c}64 \\
(53 \%)\end{array}$ & $\begin{array}{c}52 \\
(43 \%)\end{array}$ & $\begin{array}{c}2 \\
(2 \%)\end{array}$ & $\begin{array}{c}69 \\
(58 \%)\end{array}$ & $\begin{array}{c}49 \\
(41 \%)\end{array}$ & $\begin{array}{c}1 \\
(1 \%)\end{array}$ & $\begin{array}{c}59 \\
(49 \%)\end{array}$ & $\begin{array}{c}60 \\
(50 \%)\end{array}$ & $\begin{array}{c}2 \\
(2 \%)\end{array}$ & $\begin{array}{c}60 \\
(50 \%)\end{array}$ & $\begin{array}{c}58 \\
(48 \%)\end{array}$ \\
\hline 20 & $\begin{array}{l}\text { Freedom to comeback } \\
\text { from office/shopping } \\
\text { whenever the work is over }\end{array}$ & $\begin{array}{c}11 \\
(9 \%)\end{array}$ & $\begin{array}{c}61 \\
(51 \%)\end{array}$ & $\begin{array}{c}48 \\
(40 \%)\end{array}$ & $\begin{array}{c}1 \\
(1 \%)\end{array}$ & $\begin{array}{c}69 \\
(58 \%)\end{array}$ & $\begin{array}{c}50 \\
(42 \%)\end{array}$ & $\begin{array}{c}2 \\
(2 \%)\end{array}$ & $\begin{array}{c}69 \\
(58 \%)\end{array}$ & $\begin{array}{c}49 \\
(41 \%)\end{array}$ & $\begin{array}{c}2 \\
(2 \%)\end{array}$ & $\begin{array}{c}63 \\
(53 \%)\end{array}$ & $\begin{array}{c}55 \\
(46 \%)\end{array}$ \\
\hline 21 & $\begin{array}{l}\text { Freedom to grow up } \\
\text { professionally / personally }\end{array}$ & $\begin{array}{c}5 \\
(4 \%) \\
\end{array}$ & $\begin{array}{c}61 \\
(51 \%) \\
\end{array}$ & $\begin{array}{c}54 \\
(45 \%) \\
\end{array}$ & $\begin{array}{c}2 \\
(2 \%) \\
\end{array}$ & $\begin{array}{c}50 \\
(42 \%) \\
\end{array}$ & $\begin{array}{c}68 \\
(57 \%) \\
\end{array}$ & $\begin{array}{c}4 \\
(3 \%) \\
\end{array}$ & $\begin{array}{c}68 \\
(57 \%) \\
\end{array}$ & $\begin{array}{c}48 \\
(40 \%) \\
\end{array}$ & $\begin{array}{c}6 \\
(5 \%) \\
\end{array}$ & $\begin{array}{c}65 \\
(54 \%) \\
\end{array}$ & $\begin{array}{c}49 \\
(41 \%) \\
\end{array}$ \\
\hline 22 & $\begin{array}{l}\text { Freedom to leave the } \\
\text { place on transfer/holiday } \\
\text { trip/religious trip }\end{array}$ & $\begin{array}{c}5 \\
(4 \%)\end{array}$ & $\begin{array}{c}57 \\
(48 \%)\end{array}$ & $\begin{array}{c}58 \\
(48 \%)\end{array}$ & $\begin{array}{c}4 \\
(3 \%)\end{array}$ & $\begin{array}{c}66 \\
(55 \%)\end{array}$ & $\begin{array}{c}50 \\
(42 \%)\end{array}$ & $\begin{array}{c}4 \\
(3 \%)\end{array}$ & $\begin{array}{c}66 \\
(55 \%)\end{array}$ & $\begin{array}{c}50 \\
(42 \%)\end{array}$ & $\begin{array}{c}6 \\
(5 \%)\end{array}$ & $\begin{array}{c}69 \\
(58 \%)\end{array}$ & $\begin{array}{c}45 \\
(38 \%)\end{array}$ \\
\hline 23 & $\begin{array}{l}\text { Freedom to decide on } \\
\text { your role/ role } \\
\text { performance }\end{array}$ & $\begin{array}{c}4 \\
(3 \%)\end{array}$ & $\begin{array}{c}70 \\
(58 \%)\end{array}$ & $\begin{array}{c}46 \\
(38 \%)\end{array}$ & $\begin{array}{c}3 \\
(3 \%)\end{array}$ & $\begin{array}{c}68 \\
(57 \%)\end{array}$ & $\begin{array}{c}49 \\
(41 \%)\end{array}$ & $\begin{array}{c}2 \\
(2 \%)\end{array}$ & $\begin{array}{c}62 \\
(52 \%)\end{array}$ & $\begin{array}{c}56 \\
(47 \%)\end{array}$ & $\begin{array}{c}4 \\
(3 \%)\end{array}$ & $\begin{array}{c}64 \\
(53 \%)\end{array}$ & $\begin{array}{c}52 \\
(43 \%)\end{array}$ \\
\hline 24 & $\begin{array}{l}\text { Freedom to watch } \\
\text { television whenever you } \\
\text { want }\end{array}$ & $\begin{array}{c}3 \\
(3 \%)\end{array}$ & $\begin{array}{c}58 \\
(48 \%)\end{array}$ & $\begin{array}{c}59 \\
(49 \%)\end{array}$ & $\begin{array}{c}5 \\
(4 \%)\end{array}$ & $\begin{array}{c}59 \\
(49 \%)\end{array}$ & $\begin{array}{c}56 \\
(47 \%)\end{array}$ & $\begin{array}{c}4 \\
(3 \%)\end{array}$ & $\begin{array}{c}63 \\
(53 \%)\end{array}$ & $\begin{array}{c}53 \\
(44 \%)\end{array}$ & $\begin{array}{c}4 \\
(3 \%)\end{array}$ & $\begin{array}{c}67 \\
(56 \%)\end{array}$ & $\begin{array}{c}49 \\
(41 \%)\end{array}$ \\
\hline 25 & $\begin{array}{l}\text { Freedom to choose one's } \\
\text { own leisure time activity }\end{array}$ & $\begin{array}{c}2 \\
(2 \%) \\
\end{array}$ & $\begin{array}{c}59 \\
(49 \%) \\
\end{array}$ & $\begin{array}{c}59 \\
(49 \%) \\
\end{array}$ & $\begin{array}{c}4 \\
(3 \%) \\
\end{array}$ & $\begin{array}{c}69 \\
(58 \%) \\
\end{array}$ & $\begin{array}{c}47 \\
(39 \%) \\
\end{array}$ & $\begin{array}{c}7 \\
(6 \%) \\
\end{array}$ & $\begin{array}{c}66 \\
(55 \%) \\
\end{array}$ & $\begin{array}{c}47 \\
(39 \%) \\
\end{array}$ & $\begin{array}{c}5 \\
(4 \%) \\
\end{array}$ & $\begin{array}{c}59 \\
(49 \%) \\
\end{array}$ & $\begin{array}{c}56 \\
(47 \%) \\
\end{array}$ \\
\hline 26 & $\begin{array}{l}\text { Buying cosmetics / fancy } \\
\text { things }\end{array}$ & $\begin{array}{c}5 \\
(4 \%) \\
\end{array}$ & $\begin{array}{c}63 \\
(53 \%) \\
\end{array}$ & $\begin{array}{c}52 \\
(43 \%) \\
\end{array}$ & $\begin{array}{c}3 \\
(3 \%)\end{array}$ & $\begin{array}{c}63 \\
(53 \%) \\
\end{array}$ & $\begin{array}{c}54 \\
(45 \%) \\
\end{array}$ & $\begin{array}{c}6 \\
(5 \%) \\
\end{array}$ & $\begin{array}{c}65 \\
(54 \%) \\
\end{array}$ & $\begin{array}{c}49 \\
(41 \%) \\
\end{array}$ & $\begin{array}{c}2 \\
(2 \%)\end{array}$ & $\begin{array}{c}69 \\
(58 \%) \\
\end{array}$ & $\begin{array}{c}49 \\
(41 \%) \\
\end{array}$ \\
\hline 27 & $\begin{array}{l}\text { Spending on own } \\
\text { transport }\end{array}$ & $\begin{array}{c}3 \\
(3 \%)\end{array}$ & $\begin{array}{c}71 \\
(59 \%)\end{array}$ & $\begin{array}{c}46 \\
(38 \%)\end{array}$ & $\begin{array}{c}4 \\
(3 \%)\end{array}$ & $\begin{array}{c}62 \\
(52 \%)\end{array}$ & $\begin{array}{c}54 \\
(45 \%)\end{array}$ & $\begin{array}{c}6 \\
(5 \%)\end{array}$ & $\begin{array}{c}59 \\
(49 \%)\end{array}$ & $\begin{array}{c}55 \\
(46 \%) \\
\end{array}$ & $\begin{array}{c}4 \\
(3 \%)\end{array}$ & $\begin{array}{c}59 \\
(49 \%)\end{array}$ & $\begin{array}{c}57 \\
(48 \%) \\
\end{array}$ \\
\hline 28 & $\begin{array}{l}\text { Participating in social } \\
\text { gatherings }\end{array}$ & $\begin{array}{c}6 \\
(5 \%) \\
\end{array}$ & $\begin{array}{c}57 \\
(48 \%)\end{array}$ & $\begin{array}{c}57 \\
(48 \%)\end{array}$ & $\begin{array}{c}3 \\
(3 \%)\end{array}$ & $\begin{array}{c}74 \\
(62 \%)\end{array}$ & $\begin{array}{c}43 \\
(36 \%)\end{array}$ & $\begin{array}{c}3 \\
(3 \%)\end{array}$ & $\begin{array}{c}75 \\
(63 \%) \\
\end{array}$ & $\begin{array}{c}42 \\
(35 \%)\end{array}$ & $\begin{array}{c}3 \\
(3 \%)\end{array}$ & $\begin{array}{c}59 \\
(49 \%)\end{array}$ & $\begin{array}{c}58 \\
(48 \%)\end{array}$ \\
\hline 29 & $\begin{array}{l}\text { Going for higher } \\
\text { education/training }\end{array}$ & $\begin{array}{c}7 \\
(6 \%)\end{array}$ & $\begin{array}{c}65 \\
(54 \%)\end{array}$ & $\begin{array}{c}48 \\
(40 \%)\end{array}$ & $\begin{array}{c}2 \\
2 \%)\end{array}$ & $\begin{array}{c}66 \\
(55 \%)\end{array}$ & $\begin{array}{c}52 \\
(43 \%)\end{array}$ & $\begin{array}{c}2 \\
(2 \%)\end{array}$ & $\begin{array}{c}67 \\
(56 \%)\end{array}$ & $\begin{array}{c}51 \\
(43 \%)\end{array}$ & $\begin{array}{c}4 \\
(3 \%)\end{array}$ & $\begin{array}{c}64 \\
(53 \%)\end{array}$ & $\begin{array}{c}52 \\
(43 \%)\end{array}$ \\
\hline 30 & $\begin{array}{l}\text { Freedom to donate one's } \\
\text { own money }\end{array}$ & $\begin{array}{c}6 \\
(5 \%) \\
\end{array}$ & $\begin{array}{c}60 \\
(50 \%) \\
\end{array}$ & $\begin{array}{c}54 \\
(45 \%) \\
\end{array}$ & $\begin{array}{c}2 \\
(2 \%) \\
\end{array}$ & $\begin{array}{c}61 \\
(51 \%) \\
\end{array}$ & $\begin{array}{c}57 \\
(48 \%) \\
\end{array}$ & $\begin{array}{c}5 \\
(4 \%) \\
\end{array}$ & $\begin{array}{c}62 \\
(52 \%) \\
\end{array}$ & $\begin{array}{c}53 \\
(44 \%) \\
\end{array}$ & $\begin{array}{c}7 \\
(6 \%) \\
\end{array}$ & $\begin{array}{c}68 \\
(57 \%) \\
\end{array}$ & $\begin{array}{c}45 \\
(38 \%) \\
\end{array}$ \\
\hline 31 & $\begin{array}{l}\text { Choose to work outside } \\
\text { the home if necessary / } \\
\text { shift job if needed }\end{array}$ & $\begin{array}{c}6 \\
(5 \%)\end{array}$ & $\begin{array}{c}56 \\
(47 \%)\end{array}$ & $\begin{array}{c}58 \\
(48 \%)\end{array}$ & $\begin{array}{c}3 \\
(3 \%)\end{array}$ & $\begin{array}{c}72 \\
(60 \%)\end{array}$ & $\begin{array}{c}45 \\
(38 \%)\end{array}$ & $\begin{array}{c}4 \\
(3 \%)\end{array}$ & $\begin{array}{c}63 \\
(53 \%)\end{array}$ & $\begin{array}{c}53 \\
(44 \%)\end{array}$ & $\begin{array}{c}6 \\
(5 \%)\end{array}$ & $\begin{array}{c}63 \\
(53 \%)\end{array}$ & $\begin{array}{c}51 \\
(43 \%)\end{array}$ \\
\hline
\end{tabular}


According to resource availability, it is evident from Table 1 that more than half of the respondents sometimes had freedom to pursue own religious activities (74\%), personal movement to shorter distance (64\%), spending on own transport (59\%), freedom to decide on his/her role/role performance (58\%) and freedom to pursue own hobbies. Less than half of the respondents sometimes had taken self decisions on personal affairs were buying sarees/dress (27\%), personal movement to longer distances (36\%), freedom to spend own money $(45 \%)$, freedom to project own personality in a group and choose to work outside the home if necessary/shift job is needed (47\%).

With regard to access to resource, majority of the respondents was found to be sometimes freedom to pursue own religious activities (76\%), personal movement to shorter distances (65\%), Participating in social gatherings (62\%), Choose to work outside the home if necessary / shift job if needed (60\%), freedom to pursue own hobbies (59\%), freedom to concentrate on office work/personal work wholly, freedom to comeback from office/shopping whenever the work is over and Freedom to choose one's own leisure time activity (58\%), invites friends home and Freedom to decide on your role/ role performance (57\%). Sometimes least percentage of the respondents had taken self decisions on personal movement to longer distances (35\%), buying sarees/dresses (41\%), Freedom to grow up professionally / personally (42\%), freedom to attend social functions (45\%), freedom to express emotions freely, freedom to project own personality in a group freedom to spend own money (48\%), spacing the children, freedom to use contraceptive according to own choice, freedom to save money according to own choice, freedom to watch television whenever you want (49\%).

As per decision making, maximum percentage of respondents was found, sometimes freedom to pursue own religious activities (75\%), participating in social gatherings (63\%), freedom to pursue own hobbies (59\%), freedom to use contraceptive according to own choice and Freedom to comeback from office/shopping whenever the work is over (58\%), Buying less expensive and expensive jewellery, freedom to grow up professionally / personally (57\%). Minimum percentage of respondents was found sometimes personal movement to longer distance (30\%), buying sarees/dresses (32\%), Planning for no. of children (46\%), spacing the children (47\%) and freedom to spend own money, inviting friends home, save money according to own choice and having control of one's own time (48\%).

With respect to action in personal affairs, highest percentage of respondents was found sometimes freedom to pursue own religious activities (73\%), personal movement to shorter distance (62\%). Fifty eight percent of the respondents found sometimes freedom to pursue own hobbies, buying expensive jewellery, freedom to attend social functions, having control of one's own time, freedom to leave the place on transfer/holiday trip/religious trip, buying cosmetics / fancy things. Lowest percentage of respondents had taken sometimes decisions on personal Movement to longer distances (33\%), buying sarees/dress (43\%), freedom to spend own money (47\%), freedom to choose one's own leisure time activity, can do things according to one's own interests (48\%) and spending on own transport, participating in social gatherings (49\%). 
Table 2: Percentage Distribution of Respondents According to self Decisions on Children's Affairs

\begin{tabular}{|c|c|c|c|c|c|c|c|c|c|c|c|c|c|}
\hline \multirow{2}{*}{$\begin{array}{l}\text { S. } \\
\text { No } \\
\text {. }\end{array}$} & \multirow{2}{*}{ Items } & \multicolumn{3}{|c|}{ Resource Available } & \multicolumn{3}{|c|}{ Access to resource } & \multicolumn{3}{|c|}{ Decision making } & \multicolumn{3}{|c|}{ Action } \\
\hline & & $\mathbf{A}$ & $\mathbf{S}$ & $\mathbf{N}$ & $\mathbf{A}$ & $\mathbf{S}$ & $\mathbf{N}$ & $\mathbf{A}$ & $\mathbf{S}$ & $\mathbf{N}$ & $\mathbf{A}$ & $\mathbf{S}$ & $\mathbf{N}$ \\
\hline 1 & $\begin{array}{l}\text { Buying school } \\
\text { requirements }\end{array}$ & $\begin{array}{c}4 \\
(3 \%)\end{array}$ & $\begin{array}{c}68 \\
(57 \%) \\
\end{array}$ & $\begin{array}{c}48 \\
(40 \%)\end{array}$ & $\begin{array}{c}5 \\
(4 \%)\end{array}$ & $\begin{array}{c}67 \\
(56 \%) \\
\end{array}$ & $\begin{array}{c}48 \\
(40 \%) \\
\end{array}$ & $\begin{array}{c}2 \\
(2 \%)\end{array}$ & $\begin{array}{c}63 \\
(53 \%) \\
\end{array}$ & $\begin{array}{c}55 \\
(46 \%) \\
\end{array}$ & $\begin{array}{c}7 \\
(6 \%)\end{array}$ & $\begin{array}{c}65 \\
(54 \%) \\
\end{array}$ & $\begin{array}{c}48 \\
(40 \%)\end{array}$ \\
\hline 2 & $\begin{array}{l}\text { Buying clothes } \\
\text { for children }\end{array}$ & $\begin{array}{c}6 \\
(5 \%)\end{array}$ & $\begin{array}{c}95 \\
(79 \%) \\
\end{array}$ & $\begin{array}{c}19 \\
(16 \%)\end{array}$ & -- & $\begin{array}{c}87 \\
(73 \%) \\
\end{array}$ & $\begin{array}{c}33 \\
(28 \%) \\
\end{array}$ & $\begin{array}{c}2 \\
(2 \%)\end{array}$ & $\begin{array}{c}87 \\
(73 \%) \\
\end{array}$ & $\begin{array}{c}31 \\
(26 \%) \\
\end{array}$ & $\begin{array}{c}2 \\
(2 \%)\end{array}$ & $\begin{array}{c}95 \\
(79 \%) \\
\end{array}$ & $\begin{array}{c}23 \\
(19 \%)\end{array}$ \\
\hline 3 & $\begin{array}{l}\text { Freedom to } \\
\text { discipline the } \\
\text { children }\end{array}$ & $\begin{array}{c}1 \\
(1 \%)\end{array}$ & $\begin{array}{c}69 \\
(58 \%)\end{array}$ & $\begin{array}{c}50 \\
(42 \%)\end{array}$ & $\begin{array}{c}1 \\
(1 \%)\end{array}$ & $\begin{array}{c}62 \\
(52 \%)\end{array}$ & $\begin{array}{c}57 \\
(48 \%)\end{array}$ & $\begin{array}{c}3 \\
(3 \%)\end{array}$ & $\begin{array}{c}64 \\
(53 \%)\end{array}$ & $\begin{array}{c}53 \\
(44 \%)\end{array}$ & $\begin{array}{c}2 \\
(2 \%)\end{array}$ & $\begin{array}{c}70 \\
(58 \%)\end{array}$ & $\begin{array}{c}48 \\
(40 \%)\end{array}$ \\
\hline 4 & $\begin{array}{l}\text { Power to } \\
\text { inculcate values } \\
\text { in children }\end{array}$ & $\begin{array}{c}2 \\
(2 \%)\end{array}$ & $\begin{array}{c}39 \\
(33 \%)\end{array}$ & $\begin{array}{c}79 \\
(66 \%)\end{array}$ & $\begin{array}{c}3 \\
(3 \%)\end{array}$ & $\begin{array}{c}42 \\
(35 \%)\end{array}$ & $\begin{array}{c}75 \\
(63 \%)\end{array}$ & $\begin{array}{c}2 \\
(2 \%)\end{array}$ & $\begin{array}{c}50 \\
(42 \%)\end{array}$ & $\begin{array}{c}68 \\
(57 \%)\end{array}$ & $\begin{array}{c}3 \\
(3 \%)\end{array}$ & $\begin{array}{c}40 \\
(33 \%)\end{array}$ & $\begin{array}{c}77 \\
(64 \%)\end{array}$ \\
\hline 5 & $\begin{array}{l}\text { Bring up the } \\
\text { children the } \\
\text { way you want }\end{array}$ & $\begin{array}{c}5 \\
(4 \%)\end{array}$ & $\begin{array}{c}65 \\
(54 \%)\end{array}$ & $\begin{array}{c}50 \\
(42 \%)\end{array}$ & $\begin{array}{c}5 \\
(4 \%)\end{array}$ & $\begin{array}{c}71 \\
(59 \%)\end{array}$ & $\begin{array}{c}44 \\
(37 \%)\end{array}$ & $\begin{array}{c}1 \\
(1 \%)\end{array}$ & $\begin{array}{c}73 \\
(61 \%)\end{array}$ & $\begin{array}{c}46 \\
(38 \%)\end{array}$ & $\begin{array}{c}2 \\
(2 \%)\end{array}$ & $\begin{array}{c}69 \\
(58 \%)\end{array}$ & $\begin{array}{c}49 \\
(41 \%)\end{array}$ \\
\hline 6 & $\begin{array}{l}\text { Power to attend } \\
\text { children's needs } \\
\text { at school }\end{array}$ & $\begin{array}{c}4 \\
(3 \%)\end{array}$ & $\begin{array}{c}78 \\
(65 \%)\end{array}$ & $\begin{array}{c}38 \\
(32 \%)\end{array}$ & $\begin{array}{c}3 \\
(3 \%)\end{array}$ & $\begin{array}{c}69 \\
(58 \%)\end{array}$ & $\begin{array}{c}48 \\
(40 \%)\end{array}$ & $\begin{array}{c}2 \\
(2 \%)\end{array}$ & $\begin{array}{c}58 \\
(48 \%)\end{array}$ & $\begin{array}{c}60 \\
(50 \%)\end{array}$ & -- & $\begin{array}{c}70 \\
(58 \%)\end{array}$ & $\begin{array}{c}50 \\
(42 \%)\end{array}$ \\
\hline 7 & $\begin{array}{l}\text { Encourage } \\
\text { children to } \\
\text { pursue different } \\
\text { hobbies }\end{array}$ & $\begin{array}{c}3 \\
(3 \%)\end{array}$ & $\begin{array}{c}54 \\
(45 \%)\end{array}$ & $\begin{array}{c}63 \\
(53 \%)\end{array}$ & $\begin{array}{c}3 \\
(3 \%)\end{array}$ & $\begin{array}{c}47 \\
(39 \%)\end{array}$ & $\begin{array}{c}70 \\
(58 \%)\end{array}$ & $\begin{array}{c}2 \\
(2 \%)\end{array}$ & $\begin{array}{c}70 \\
(58 \%)\end{array}$ & $\begin{array}{c}48 \\
(40 \%)\end{array}$ & $\begin{array}{c}4 \\
(3 \%)\end{array}$ & $\begin{array}{c}64 \\
(53 \%)\end{array}$ & $\begin{array}{c}52 \\
(43 \%)\end{array}$ \\
\hline 8 & $\begin{array}{l}\text { Bring up the } \\
\text { children the } \\
\text { way you want }\end{array}$ & $\begin{array}{c}5 \\
(4 \%)\end{array}$ & $\begin{array}{c}65 \\
(54 \%)\end{array}$ & $\begin{array}{c}50 \\
(42 \%)\end{array}$ & $\begin{array}{c}5 \\
(4 \%)\end{array}$ & $\begin{array}{c}71 \\
(59 \%)\end{array}$ & $\begin{array}{c}44 \\
(37 \%)\end{array}$ & $\begin{array}{c}1 \\
(1 \%)\end{array}$ & $\begin{array}{c}73 \\
(61 \%)\end{array}$ & $\begin{array}{c}46 \\
(38 \%)\end{array}$ & $\begin{array}{c}2 \\
(2 \%)\end{array}$ & $\begin{array}{c}69 \\
(58 \%)\end{array}$ & $\begin{array}{c}49 \\
(41 \%)\end{array}$ \\
\hline 9 & $\begin{array}{l}\text { Buying minor } \\
\text { things for } \\
\text { children }\end{array}$ & $\begin{array}{c}2 \\
(2 \%)\end{array}$ & $\begin{array}{c}53 \\
(44 \%)\end{array}$ & $\begin{array}{c}65 \\
(54 \%)\end{array}$ & $\begin{array}{c}3 \\
(3 \%)\end{array}$ & $\begin{array}{c}60 \\
(50 \%)\end{array}$ & $\begin{array}{c}57 \\
(48 \%)\end{array}$ & $\begin{array}{c}2 \\
(2 \%)\end{array}$ & $\begin{array}{c}76 \\
(63 \%)\end{array}$ & $\begin{array}{c}42 \\
(35 \%)\end{array}$ & $\begin{array}{c}2 \\
(2 \%)\end{array}$ & $\begin{array}{c}59 \\
(49 \%)\end{array}$ & $\begin{array}{c}59 \\
(49 \%)\end{array}$ \\
\hline
\end{tabular}

It is clear from the Table 2, resource availability revealed that the respondents was found high in buying clothes for children (79\%), power to attend children's needs at school (65\%), freedom to discipline the children (58\%), buying school requirements $(57 \%)$ and bring up the children the way you want (54\%) whereas least was found sometimes in power to inculcate values in children (33\%), buying minor things for children (44\%) and encourage children to pursue different hobbies $(45 \%)$.

In access to resource was found high in buying clothes for children (73\%), bring up the children the way you want (59\%), power to attend children's need at school (58\%), buying school requirements (56\%), freedom to discipline the children $(52 \%)$ and buying minor things for children $(50 \%)$. Lowest percentage of respondents had taken sometimes self decisions on power to inculcate values in children (35\%) and encourage children to pursue different hobbies (39\%).

In decision making, highest percentage was found in buying clothes for children (73\%), buying minor things for children $(63 \%)$, bring up the children the way you want $(61 \%)$, encourage children to pursue different hobbies $(58 \%)$, buying school requirements and freedom to discipline the children $(53 \%)$. Least percentage had taken sometimes on power to inculcate values in children (42\%) and power to attend children's needs at school (48\%).

In action to children's affairs, minimum percentage was found in buying clothes for children (79\%) and minimum percentage was found in power to inculcate values in children $(33 \%)$. Equal percentage (58\%) of the respondents was found sometimes freedom to discipline the children, bringing up the children the way you want and power to attend children's needs at school. 
Table 3: Percentage Distribution of Respondents According to Self Decisions on Household Affairs

\begin{tabular}{|c|c|c|c|c|c|c|c|c|c|c|c|c|c|}
\hline \multirow{2}{*}{$\begin{array}{l}\text { S. } \\
\text { No. }\end{array}$} & \multirow{2}{*}{ Items } & \multicolumn{3}{|c|}{ Resource Available } & \multicolumn{3}{|c|}{ Access to resource } & \multicolumn{3}{|c|}{ Decision making } & \multicolumn{3}{|c|}{ Action } \\
\hline & & $\mathbf{A}$ & $\mathbf{S}$ & $\mathbf{N}$ & $\mathbf{A}$ & $\mathbf{S}$ & $\mathbf{N}$ & $\mathbf{A}$ & $\mathbf{S}$ & $\mathbf{N}$ & $\mathbf{A}$ & $\mathbf{S}$ & $\mathbf{N}$ \\
\hline 1 & $\begin{array}{l}\text { Buying } \\
\text { miscellaneous } \\
\text { things for the } \\
\text { household } \\
\text { management }\end{array}$ & $\begin{array}{c}6 \\
(5 \%)\end{array}$ & $\begin{array}{c}71 \\
(59 \%)\end{array}$ & $\begin{array}{c}43 \\
(36 \%)\end{array}$ & $\begin{array}{c}3 \\
(3 \%)\end{array}$ & $\begin{array}{c}75 \\
(63 \%)\end{array}$ & $\begin{array}{c}42 \\
(35 \%)\end{array}$ & $\begin{array}{c}2 \\
(2 \%)\end{array}$ & $\begin{array}{c}80 \\
(67 \%)\end{array}$ & $\begin{array}{c}38 \\
(32 \%)\end{array}$ & $\begin{array}{c}4 \\
(3 \%)\end{array}$ & $\begin{array}{c}67 \\
(56 \%)\end{array}$ & $\begin{array}{c}49 \\
(41 \%)\end{array}$ \\
\hline 2 & $\begin{array}{l}\text { Decorating the } \\
\text { house according } \\
\text { to own choice }\end{array}$ & $\begin{array}{c}2 \\
(2 \%)\end{array}$ & $\begin{array}{c}82 \\
(68 \%)\end{array}$ & $\begin{array}{c}36 \\
(30 \%)\end{array}$ & $\begin{array}{c}4 \\
(3 \%)\end{array}$ & $\begin{array}{c}86 \\
(72 \%)\end{array}$ & $\begin{array}{c}30 \\
(25 \%)\end{array}$ & $\begin{array}{c}3 \\
(3 \%)\end{array}$ & $\begin{array}{c}87 \\
(73 \%)\end{array}$ & $\begin{array}{c}30 \\
(25 \%)\end{array}$ & $\begin{array}{c}3 \\
(3 \%)\end{array}$ & $\begin{array}{c}81 \\
(68 \%)\end{array}$ & $\begin{array}{c}36 \\
(30 \%)\end{array}$ \\
\hline 3 & $\begin{array}{l}\text { Engaging the paid } \\
\text { help according to } \\
\text { own choice }\end{array}$ & $\begin{array}{c}4 \\
(3 \%)\end{array}$ & $\begin{array}{c}82 \\
(68 \%)\end{array}$ & $\begin{array}{c}34 \\
(28 \%)\end{array}$ & $\begin{array}{c}5 \\
(4 \%)\end{array}$ & $\begin{array}{c}86 \\
(72 \%)\end{array}$ & $\begin{array}{c}29 \\
(24 \%)\end{array}$ & $\begin{array}{c}2 \\
(2 \%)\end{array}$ & $\begin{array}{c}78 \\
(65 \%)\end{array}$ & $\begin{array}{c}40 \\
(33 \%)\end{array}$ & $\begin{array}{c}6 \\
(5 \%)\end{array}$ & $\begin{array}{c}71 \\
(59 \%)\end{array}$ & $\begin{array}{c}43 \\
(36 \%)\end{array}$ \\
\hline 4 & $\begin{array}{l}\text { Keeping parents } \\
\text { or relatives at } \\
\text { home }\end{array}$ & -- & $\begin{array}{c}52 \\
(43 \%)\end{array}$ & $\begin{array}{c}68 \\
(57 \%)\end{array}$ & $\begin{array}{c}4 \\
(3 \%)\end{array}$ & $\begin{array}{c}38 \\
(32 \%)\end{array}$ & $\begin{array}{c}78 \\
(65 \%)\end{array}$ & -- & $\begin{array}{c}46 \\
(38 \%)\end{array}$ & $\begin{array}{c}74 \\
(62 \%)\end{array}$ & $\begin{array}{c}3 \\
(3 \%)\end{array}$ & $\begin{array}{c}44 \\
(37 \%)\end{array}$ & $\begin{array}{c}73 \\
(61 \%)\end{array}$ \\
\hline 5 & $\begin{array}{l}\text { Freedom to } \\
\text { choose to live } \\
\text { with in-laws or } \\
\text { without in-laws }\end{array}$ & $\begin{array}{c}2 \\
(2 \%)\end{array}$ & $\begin{array}{c}61 \\
(51 \%)\end{array}$ & $\begin{array}{c}57 \\
(48 \%)\end{array}$ & $\begin{array}{c}3 \\
(3 \%)\end{array}$ & $\begin{array}{c}40 \\
(33 \%)\end{array}$ & $\begin{array}{c}77 \\
(64 \%)\end{array}$ & $\begin{array}{c}1 \\
(1 \%)\end{array}$ & $\begin{array}{c}49 \\
(41 \%)\end{array}$ & $\begin{array}{c}70 \\
(58 \%)\end{array}$ & $\begin{array}{c}4 \\
(3 \%)\end{array}$ & $\begin{array}{c}44 \\
(37 \%)\end{array}$ & $\begin{array}{c}72 \\
(60 \%)\end{array}$ \\
\hline 6 & $\begin{array}{l}\text { Maintain the } \\
\text { household } \\
\text { according to own } \\
\text { choice }\end{array}$ & $\begin{array}{c}5 \\
(4 \%)\end{array}$ & $\begin{array}{c}64 \\
(53 \%)\end{array}$ & $\begin{array}{c}51 \\
(43 \%)\end{array}$ & $\begin{array}{c}2 \\
(2 \%)\end{array}$ & $\begin{array}{c}72 \\
(60 \%)\end{array}$ & $\begin{array}{c}46 \\
(38 \%)\end{array}$ & $\begin{array}{c}1 \\
(1 \%)\end{array}$ & $\begin{array}{c}69 \\
(58 \%)\end{array}$ & $\begin{array}{c}50 \\
(42 \%)\end{array}$ & $\begin{array}{c}3 \\
(3 \%)\end{array}$ & $\begin{array}{c}70 \\
(58 \%)\end{array}$ & $\begin{array}{c}47 \\
(39 \%)\end{array}$ \\
\hline 7 & $\begin{array}{l}\text { Engaging friends } \\
\text { at home }\end{array}$ & $\begin{array}{c}2 \\
(2 \%)\end{array}$ & $\begin{array}{c}69 \\
(58 \%)\end{array}$ & $\begin{array}{c}49 \\
(41 \%)\end{array}$ & $\begin{array}{c}1 \\
(1 \%)\end{array}$ & $\begin{array}{c}74 \\
(62 \%)\end{array}$ & $\begin{array}{c}45 \\
(38 \%)\end{array}$ & $\begin{array}{c}2 \\
(2 \%)\end{array}$ & $\begin{array}{c}67 \\
(56 \%)\end{array}$ & $\begin{array}{c}51 \\
(43 \%)\end{array}$ & $\begin{array}{c}2 \\
(2 \%)\end{array}$ & $\begin{array}{c}68 \\
(57 \%)\end{array}$ & $\begin{array}{c}50 \\
(42 \%) \\
\end{array}$ \\
\hline 8 & $\begin{array}{l}\text { Supporting natal } \\
\text { family through } \\
\text { kind }\end{array}$ & $\begin{array}{c}2 \\
(2 \%)\end{array}$ & $\begin{array}{c}59 \\
(49 \%)\end{array}$ & $\begin{array}{c}59 \\
(49 \%)\end{array}$ & $\begin{array}{c}3 \\
(3 \%)\end{array}$ & $\begin{array}{c}66 \\
(55 \%)\end{array}$ & $\begin{array}{c}51 \\
(43 \%)\end{array}$ & $\begin{array}{c}1 \\
(1 \%)\end{array}$ & $\begin{array}{c}73 \\
(61 \%)\end{array}$ & $\begin{array}{c}46 \\
(38 \%)\end{array}$ & $\begin{array}{c}3 \\
(3 \%)\end{array}$ & $\begin{array}{c}63 \\
(53 \%)\end{array}$ & $\begin{array}{c}54 \\
(45 \%)\end{array}$ \\
\hline 9 & $\begin{array}{l}\text { Supporting natal } \\
\text { family financially }\end{array}$ & $\begin{array}{c}4 \\
(3 \%)\end{array}$ & $\begin{array}{c}61 \\
(51 \%)\end{array}$ & $\begin{array}{c}55 \\
(46 \%)\end{array}$ & $\begin{array}{c}5 \\
(4 \%)\end{array}$ & $\begin{array}{c}72 \\
(60 \%)\end{array}$ & $\begin{array}{c}43 \\
(36 \%)\end{array}$ & $\begin{array}{c}6 \\
(5 \%)\end{array}$ & $\begin{array}{c}68 \\
(57 \%)\end{array}$ & $\begin{array}{c}46 \\
(38 \%)\end{array}$ & $\begin{array}{c}7 \\
(6 \%)\end{array}$ & $\begin{array}{c}55 \\
(46 \%)\end{array}$ & $\begin{array}{c}58 \\
(48 \%)\end{array}$ \\
\hline 10 & $\begin{array}{l}\text { Supporting in- } \\
\text { laws through kind }\end{array}$ & $\begin{array}{c}3 \\
(3 \%) \\
\end{array}$ & $\begin{array}{c}61 \\
(51 \%)\end{array}$ & $\begin{array}{c}56 \\
(47 \%)\end{array}$ & $\begin{array}{c}2 \\
(2 \%) \\
\end{array}$ & $\begin{array}{c}62 \\
(52 \%)\end{array}$ & $\begin{array}{c}56 \\
(47 \%)\end{array}$ & $\begin{array}{c}2 \\
(2 \%)\end{array}$ & $\begin{array}{c}78 \\
(65 \%) \\
\end{array}$ & $\begin{array}{c}40 \\
(33 \%)\end{array}$ & $\begin{array}{c}2 \\
(2 \%) \\
\end{array}$ & $\begin{array}{c}67 \\
(56 \%)\end{array}$ & $\begin{array}{c}51 \\
(43 \%) \\
\end{array}$ \\
\hline 11 & $\begin{array}{l}\text { Supporting in- } \\
\text { laws family } \\
\text { financially }\end{array}$ & $\begin{array}{c}5 \\
(4 \%)\end{array}$ & $\begin{array}{c}62 \\
(52 \%)\end{array}$ & $\begin{array}{c}53 \\
(44 \%)\end{array}$ & $\begin{array}{c}7 \\
(6 \%)\end{array}$ & $\begin{array}{c}66 \\
(55 \%)\end{array}$ & $\begin{array}{c}47 \\
(39 \%)\end{array}$ & $\begin{array}{c}2 \\
(2 \%)\end{array}$ & $\begin{array}{c}60 \\
(50 \%)\end{array}$ & $\begin{array}{c}58 \\
(48 \%)\end{array}$ & $\begin{array}{c}2 \\
(2 \%)\end{array}$ & $\begin{array}{c}61 \\
(51 \%)\end{array}$ & $\begin{array}{c}57 \\
(48 \%)\end{array}$ \\
\hline 12 & $\begin{array}{l}\text { Go out of the } \\
\text { house when } \\
\text { needed for } \\
\text { shopping }\end{array}$ & $\begin{array}{c}2 \\
(2 \%)\end{array}$ & $\begin{array}{c}67 \\
(56 \%)\end{array}$ & $\begin{array}{c}51 \\
(43 \%)\end{array}$ & $\begin{array}{c}4 \\
(3 \%)\end{array}$ & $\begin{array}{c}69 \\
(58 \%)\end{array}$ & $\begin{array}{c}47 \\
(39 \%)\end{array}$ & $\begin{array}{c}3 \\
(3 \%)\end{array}$ & $\begin{array}{c}62 \\
(52 \%)\end{array}$ & $\begin{array}{c}55 \\
(46 \%)\end{array}$ & $\begin{array}{c}3 \\
(3 \%)\end{array}$ & $\begin{array}{c}68 \\
(57 \%)\end{array}$ & $\begin{array}{c}49 \\
(41 \%)\end{array}$ \\
\hline 13 & $\begin{array}{l}\text { Buying groceries } \\
\text { for the household }\end{array}$ & $\begin{array}{c}3 \\
(3 \%)\end{array}$ & $\begin{array}{c}57 \\
(48 \%)\end{array}$ & $\begin{array}{c}60 \\
(50 \%)\end{array}$ & $\begin{array}{c}2 \\
(2 \%)\end{array}$ & $\begin{array}{c}71 \\
(59 \%)\end{array}$ & $\begin{array}{c}47 \\
(39 \%)\end{array}$ & $\begin{array}{c}2 \\
(2 \%)\end{array}$ & $\begin{array}{c}85 \\
(71 \%)\end{array}$ & $\begin{array}{c}33 \\
(28 \%)\end{array}$ & $\begin{array}{c}2 \\
(2 \%)\end{array}$ & $\begin{array}{c}62 \\
(52 \%)\end{array}$ & $\begin{array}{c}56 \\
(47 \%)\end{array}$ \\
\hline 14 & $\begin{array}{l}\text { Buying } \\
\text { vegetables for the } \\
\text { household }\end{array}$ & $\begin{array}{c}4 \\
(3 \%)\end{array}$ & $\begin{array}{c}63 \\
(53 \%)\end{array}$ & $\begin{array}{c}53 \\
(44 \%)\end{array}$ & -- & $\begin{array}{c}70 \\
(58 \%)\end{array}$ & $\begin{array}{c}50 \\
(42 \%)\end{array}$ & $\begin{array}{c}4 \\
(3 \%)\end{array}$ & $\begin{array}{c}65 \\
(54 \%)\end{array}$ & $\begin{array}{c}51 \\
(43 \%)\end{array}$ & $\begin{array}{c}4 \\
(3 \%)\end{array}$ & $\begin{array}{c}57 \\
(48 \%)\end{array}$ & $\begin{array}{c}59 \\
(49 \%)\end{array}$ \\
\hline 15 & $\begin{array}{l}\text { Buying outside } \\
\text { food for the } \\
\text { family }\end{array}$ & $\begin{array}{c}2 \\
(2 \%)\end{array}$ & $\begin{array}{c}59 \\
(49 \%)\end{array}$ & $\begin{array}{c}59 \\
(49 \%)\end{array}$ & $\begin{array}{c}3 \\
(3 \%)\end{array}$ & $\begin{array}{c}55 \\
(46 \%)\end{array}$ & $\begin{array}{c}62 \\
(52 \%)\end{array}$ & $\begin{array}{c}2 \\
(2 \%)\end{array}$ & $\begin{array}{c}56 \\
(47 \%)\end{array}$ & $\begin{array}{c}62 \\
(52 \%)\end{array}$ & $\begin{array}{c}4 \\
(3 \%)\end{array}$ & $\begin{array}{c}53 \\
(44 \%)\end{array}$ & $\begin{array}{c}63 \\
(53 \%)\end{array}$ \\
\hline
\end{tabular}

According to resource available, majority of the respondents sometimes takes self decisions on household affairs viz., decorating the house according to own choice and engaging the paid help according to own choice (68\%), buying miscellaneous things for the household management (59\%), engaging friends at home (58\%), go out of the house when needed for shopping (56\%), maintain the household according to own choice and buying vegetables for the household (53\%), supporting in-laws family financially (52\%). Five per cent of the respondents had taken self decisions always on household affairs and four per cent of the respondents had taken self decisions always on maintain the household according to own choice and supporting in-laws family financially (Table 3 ).

As per access to resource, equal percentage of respondents sometimes (72\%) had taken decisions on decorating the house and engaging the paid help according to own choice followed by buying miscellaneous things for the household management (63\%), engaging friends at home (62\%), maintains the household according to own choice and supporting 
natal family financially (60\%). Only six per cent of the respondents had taken joint decisions always on supporting in-laws family financially (Table 3).

While in decision making, seventy three per cent of the respondents had taken decision on decorating the house according to own choice followed by buying groceries for the household (71\%), buying miscellaneous things for the household management (67\%), engaging the paid help according to own choice and supporting in-laws through kind (65\%), supporting natal family through kind $(61 \%)$. Five per cent of the respondents had taken self decisions always on supporting natal family financially (Table 3 ).

With regard to action, sixty eight per cent of the respondents had taken self decisions on decorating the house, engaging the paid help (59\%), maintains the household (58\%). Fifty seven per cent of the respondents had taken sometimes self decisions on engaging friends at home, go out of the house when needed for shopping. Six per cent of the respondents had always taken decisions on supporting natal family financially (Table 3).

\section{Major Findings}

Majority of the respondents according to self decisions on personal affairs were found high in freedom to pursue own religious activities, in resource available, access to resource, decision making and action of self decisions on personal affairs.

Nearly three-fourth of the respondents according to self decisions on children affairs were found high in buying clothes for children in resource availability and action, access to resource and decision making and less was found in power to inculcate values in children in resource availability and action, access to resource, decision making.

Majority of the respondents according to self decisions on house hold affairs had taken self decisions sometimes on decorating the house and engaging the paid help according to their own choice.

\section{CONCLUSIONS}

Findings of the study inferred that there are women who are still disempowered, suppressed by the society. Lack of education and low levels of decision making skills women might dominated by men in majority of the farm decisions in rural families. There is a need for life skills education to enhance their self decisions in their personal, child and house hold affairs aspects of farm women. These life skills education will help women be aware of their rights and have contribution to society, get employment in formal sectors, reduce poverty and help in household expenditure. Well educated women can better utilize resources for a sustainable positive change in the society.

\section{REFERENCES}

1. Baliyan, K. (2014). Factors affecting participation of woman in household decision making. Retrieved from http://www.indiastat.com/SOCIO_PDF/103/fulltext.pdf.

2. Rajesh Dahiya \& Saroj Yadav, "Economic Empowerment of Women through Skill Up Gradation”, International Journal of Humanities and Social Sciences (IJHSS) ISSN(P): 2319-393X; ISSN(E): 2319-3948 Vol. 7, Issue 3, pp; 13-18

3. Bano, T. I.-I. (2014). The women and decision making in urban India: A gender geographical study of Varanasi City, Uttar Pradesh, India. The International Journal of Engineering and Science (IJES), 3(4), 1-11.

4. Rondla Anitha, Bhoopendra Kumar Singh \& Afifa Jahan, "An Evaluation of Drudgery Reducing Agricultural Technologies Developed for Farm Women", International Journal of Agricultural Science and Research (IJASR), Vol. 9, Issue 2, pp, 35-42 
5. Basu, S., \& Basu, P. (2001). Income generation program and empowerment of women-a case study in India. Article retrieved from.

6. Neelam Basera, Neelam Bhardwaj \& Birendra Kumar, "Opinion Leadership among Farm Women in Hill Districts of Uttarakhand State, India”, International Journal of Agricultural Science and Research (IJASR), Vol. 8, Issue 6, pp, 53-58

7. Devi, M., \& Rayalu, T. (2003). Perception of working and non-working women on their relative empowerment in self decisions in urban families. Indian Psychological Review, 60(1), 10-16.

8. Bibhu Santosh Behera \& Bibhuti Prasad Mohapatra, "A Gender Comparative Socio-Economic Study With Respect to Social Participation, Membership Status and Outward Orientation among the Farmers and Farmwomen in Keonjhar District of Odisha”, International Journal of Environment, Ecology, Family and Urban Studies (IJEEFUS), Vol. 6, Issue 3, Jun 2016, 91 102

9. Gupta, K. and Yesudian, P. P. 2006. "Evidence of Women's Empowerment in India: A Study of Socio-spatial Disparities." Geojournal 65 (4).

10. Minaxi, P., \& Lopamudra, R. (2000). Role of farm women in decision making process. JADU, 10(2), 64-68.

11. Naqvi, R. H., \& Ibrar, M. (2015). Views and opinion of rural women of District Multan about their involvement and participation in family decisions: A means for women's empowerment in Pakistani society. International Journal of Social Science and Humanity, 5(2), 204-208.

12. Sharma, S., Rao, P. K., \& Sharma, R. (2013). Role of women in decision-making related to farm: A study of Jammu District of $J$ \& K State. International Journal of Scientific and Research Publications, 3(1), 1-4.

13. Sridevi, T. O. (2005). Empowerment of Women-A systematic analysis. IDF Discussion Paper retrieved from http://citeseerx.ist.psu.edu/viewdoc/download?doi=10.1.1.601.7147\& rep=rep1\&type $=$ pdf.

14. Swain, R. B., \& Wallentin, F. Y. (2009). Does microfinance empower women? Evidence from self-help groups in India. International Review of Applied Economics, 23(5), 541-556.

15. Zaman, H.2000. "Assessing the Poverty and Vulnerability Impact of Micro-Credit in Bangladesh: A case study of BRAC." World Bank Policy Research Working Paper no 2145, World Bank, Washington DC. 
